\title{
Space Charge in LLDPE Loaded with Nanoparticles
}

\author{
$\mathrm{G} \mathrm{Chen}^{1}$, C Zhang ${ }^{2}$ and G Stevens ${ }^{2}$ \\ ${ }^{1}$ School of Electronics and Computer Science \\ University of Southampton, United Kingdom \\ ${ }^{2}$ GnoSys UK Ltd, Surrey, United Kingdom
}

\begin{abstract}
Fillers are widely used in insulation systems to achieve specific electrical, mechanical and thermal properties. Recently, polymer nanocomposites have attracted significant attention as a means of improving their performance and widening their utility. To better use the nanocomposites, a thorough understanding of interaction between nanoparticles and their matrix is important. The interaction can be studied by various methods. In the present study we focus our attention on space charge dynamics of linear low density polyethylene (LLDPE) loaded with nanometric size aluminium oxide (alumina) under dc electric fields. To obtain a good dispersion of nano fillers in LLDPE, a Brabender mixer has been utilised. Four types of LLDPE nanocomposite films were prepared with concentrations of alumina of $0,1,5$ and $10 \mathrm{wt} \%$ for space charge measurements. They were electrically stressed at three electric field levels of 10,50 and $100 \mathrm{kV} / \mathrm{mm}$ and space charge dynamics during stressing period and decay after the removal of the applied electric field were observed. The results show that space charge dynamics in nanocomposites are dependent on both content of filler and the applied electric field. Generally, the amount of space charge in the sample with $1 \%$ of alumina is less while there is a significant change in characteristics in the sample loaded with 5\% alumina or more. In particular, the electrical performance in the sample with $10 \%$ alumina deteriorates, indicating there is an optimal load of alumina.
\end{abstract}

\section{INTRODUCTION}

Recently, polymer nanocomposites have attracted wide interest as a method of enhancing polymer properties and extending their utility. In polymer nanocomposites, chemically dissimilar components are combined at the nanometer scale, and stronger interactions between the polymer and nanoparticles produce markedly improved materials with better electrical, mechanical, thermal, and rheological performances than the conventional filled polymer composites. In recent years, polymer nanocomposites have been extensively studied in optical, thermal and mechanical properties, but there has been relatively little research into dielectric properties [1-4]. Considering polymers and polymer composites as traditional electrical insulating materials, but with some exceptions, few polymer nanocomposites are used in electrical insulation industry. Therefore, it is very important to investigate the dielectric properties of polymer nanocomposites.

It is well known that the interface in the composite has a significant influence on its physical properties. In polymer nanocomposites, the interfacial region between nanoparticles and the matrix has a high volume fraction because of the high surface-to-volume ratio of the fillers [1]. Therefore, polymer nanocomposites enable us to examine the effects of large internal surface (interfacial) contributions to dielectric response. On the other hand, it is also very important to understand the dielectric behaviours of polymer nanocomposites in engineering. Space charge measurement provides a powerful tool in studying polymer composites, and it is sensitive to the interfacial charges formed at the interface between two different dielectrics (Maxwell-Wagner-Sillars polarization). It is expected that space charge dynamics of the polymer composites are affected by not only the nature of the filler and matrix but also the properties of the interface which are related to the dispersion of the fillers, filler-filler and fillermatrix interactions. The role of the interface in electrical properties of the polymer nanocomposites has been investigated by some researchers. Lewis suggested that the double layer in interfacial region under the external electric field has a significant high conductivity in the lateral direction than that in the bulk, and he explained the unusual high permittivity of the filled composite with this model [1]. Nelson and $\mathrm{Hu}$ found that the space charges decay much faster in the $\mathrm{TiO}_{2}$-epoxy nanocomposite than that in $\mathrm{TiO}_{2}$-epoxy microcomposite which suggests the nanocomposite has a higher conductive interface [2]. Tanaka et al recently proposed a multi-core model to explain the some high electric field phenomena in the nanocomposites [4]. Over the years, several studies on space charge characteristics in nanocomposites have been carried out [5-7]. It has been demonstrated that the addition of nanoparticles hinder space charge formation. However the exact mechanisms why less charge is observed in nanocomposite is not clear and more experimental works are needed to build up understandings in this area.

Linear low density polyethylene (LLDPE) is a thermoplastic which is widely used as electrical insulating materials in power cable. It is a non-polar polymer and has a high viscosity in the melt phase. A nanometric size aluminium oxide powder was chosen as filler, it is a polar inorganic compound. Conventional alumina powder is commonly used as filler to improve electrical, mechanical and thermal properties in insulating composites, however, there is little in the published literature about the application of nanometric size alumina in insulating composites.

In this paper, space charge dynamics in nano Alumina filled LLDPE under various dc electric fields have been studied using the pulsed electroacoustic (PEA) technique.

\section{EXPERIMENTAL DETAILS}

\section{A. Sample Preparation}


LLDPE used in this study is a commercial linear lowdensity polyethylene from Atofina. It has a density of 0.934 $\mathrm{g} / \mathrm{cm}^{3}$, a melt index of $0.87 \mathrm{~g} / 10 \mathrm{~min}$. Nanoparticles of aluminium oxide from Degussa with a particle size of about $13 \mathrm{~nm}$ were used as filler. This nano scale filler has a nearly spherical shape with a specific surface area of about 100 $\mathrm{m}^{2} / \mathrm{g}$. The filler was dried before using. Polyethylene nanocomposites were prepared by melt mixing at $150{ }^{\circ} \mathrm{C}$ using a Brabender W50EHT mixer with chamber size of 50 $\mathrm{cm}^{3}$ under a nitrogen atmosphere. The mixer has a high shear force, the screw speed was controlled at $60 \mathrm{rpm}$, and the mixing time was $20 \mathrm{~min}$ for each sample. The sheets of mixtures were then extruded by using a Polylab Universal Moulding Machine at $150{ }^{\circ} \mathrm{C}$. The nanocomposites were finally prepared into films with the thickness of $0.2 \mathrm{~mm}$ by hot melt pressing at $150{ }^{\circ} \mathrm{C}$ or $180{ }^{\circ} \mathrm{C}$. Four types of polyethylene nanocomposite films were prepared with concentrations of alumina of $0,1,5$, and $10 \mathrm{wt} \%$, respectively. For $10 \%$ the moulding temperature has to be raised to $180{ }^{\circ} \mathrm{C}$ to achieve a uniform sample. Table 1 shows the details of the samples prepared.

Table 1 Sample details

\begin{tabular}{|c|c|c|}
\hline sample & $\%$ of alumina & moulding temperatures $\left({ }^{\circ} \mathrm{C}\right)$ \\
\hline PE-0 & 0 & 150 \\
\hline PE-1 & 1 & 150 \\
\hline PE-5 & 5 & 150 \\
\hline PE-10 & 10 & 180 \\
\hline
\end{tabular}

\section{B. PEA system}

PEA technique utilises the interaction between charge layers and an externally applied voltage pulse (in orders of nsec), to create acoustic pressure waves proportional to the charge layers based on Lorentz's Law [8]. These acoustic pressure waves are converted into voltage signal with a piezoelectric transducer. As the voltage output from the piezoelectric transducer is small, it is necessary to amplify signal with an amplifier before being captured by an oscilloscope. The princople of the PEA and a typical output from an oscilloscope is shown in Figure 1. To convert the signal Vs(t) into space charge information across the sample a technique known as deconvolution [9] is required.

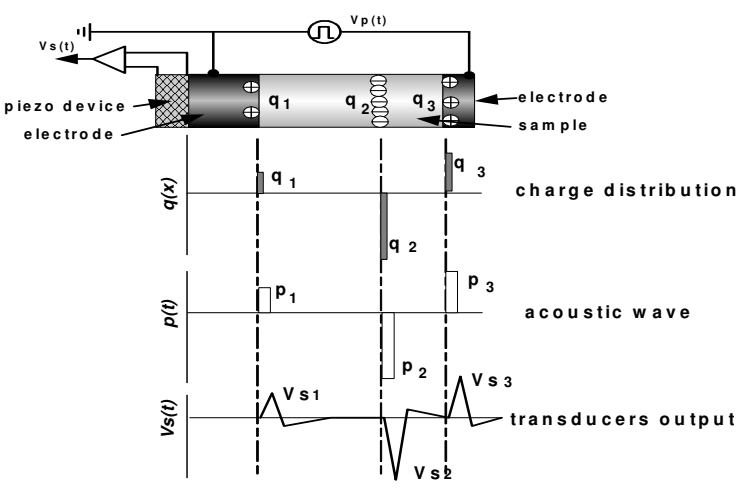

Figure 1 Schematic diagram showing the principle of PEA.

\section{Voltage Profile}

The voltage was applied for a typically 60 minutes and space charge measurements were taken at various times. To have a clear picture of the charge formation the external voltage was switched off and space charge formed in the sample were measured prior to the voltage switching back on. The sample was short-circuited upon the removal of the external voltage and the charge decay was monitored for 30 minutes. Three voltage levels were used and they were $2 \mathrm{kV}, 5$ $\mathrm{kV}$ and $10 \mathrm{kV}$ which gave applied electric fields around 10 $\mathrm{kV} / \mathrm{mm}, 25 \mathrm{kV} / \mathrm{mm}$ and $50 \mathrm{kV} / \mathrm{mm}$ respectively. A typical voltage profile for $5 \mathrm{kV}$ and various measurement points are shown in Figure 2.

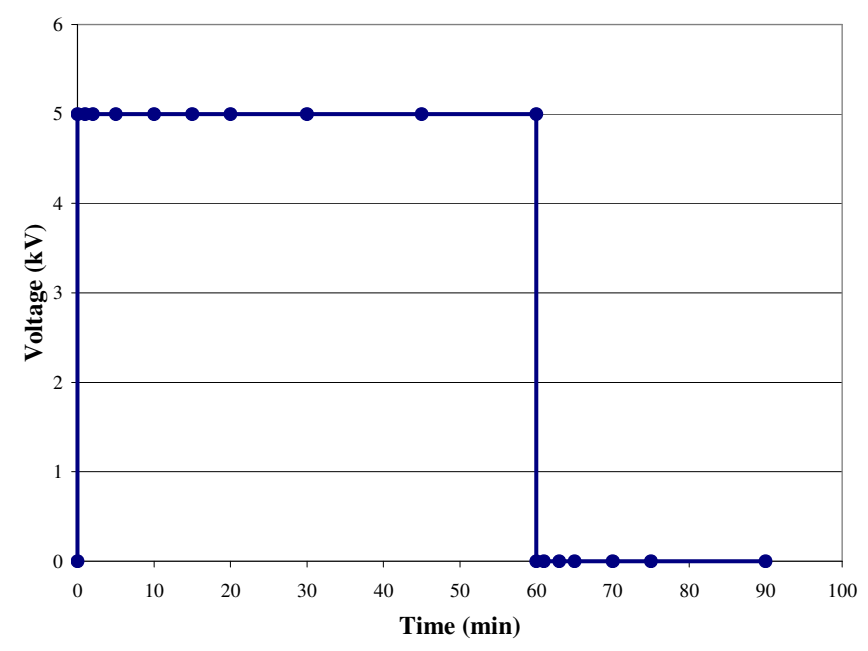

Figure 2 Voltage profile at $5 \mathrm{kV}$ and PEA measurements points.

\section{EXPERIMENTAL RESULTS AND DISCUSSION}

To reveal charge formation in the sample the results from 'volts-off' measurements have been used in the present paper. 'Volts-off' measurements are measurements taken immediately after the removal of the applied voltage. However, due to limits on paper length charge decay results will not be presented and discussed in the present paper.

Generally, at a voltage of $2 \mathrm{kV}$ there is less charge formed in the sample. Charge injection has been observed but the amount of charge is generally small in samples containing $0 \%$ and $1 \%$ alumina. When the amount of alumina is increased to $5 \%$, significant amount of charge has been observed as shown in Figure 3 (a). The two electrode positions are also illustrated and they should be at the same positions in all the rest figures. It can be seen that the amount of charge in the sample increases with the duration of the applied voltage. Charges are dominated by hetrocharges, meaning ionisation process occurred in the sample. Considering charge injection has taken place in samples without or with low percentage alumina, charge injection may also take place in the present case. The 
source of ionisation may be related to impurities of alumina. As mentioned before the amount of charge injection is small therefore it may be covered by ionisation. Actually, the injection can be seen in the region adjacent to the anode at a later stage of the stressing period as indicated by an arrow in figure. The broad peak indicates the positive charge close to the anode.

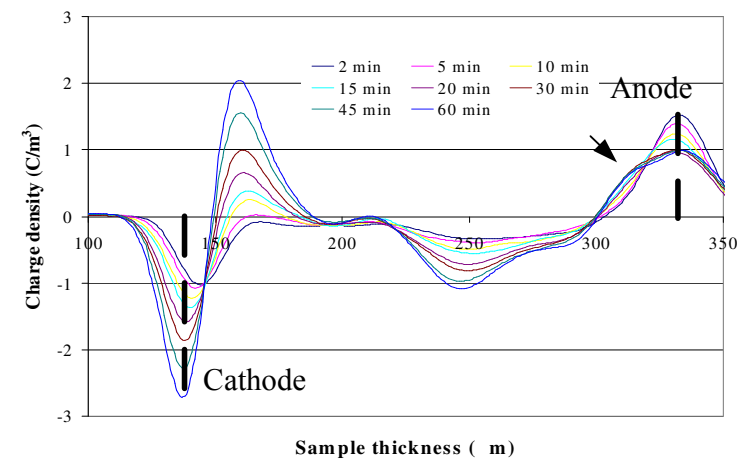

(a) PE-5

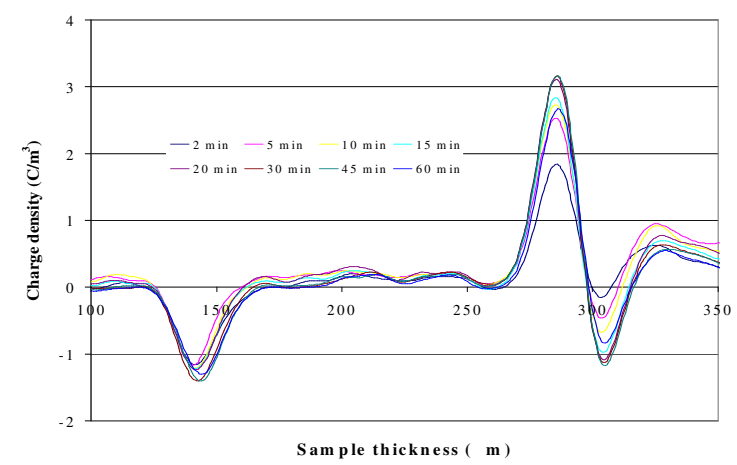

(b) PE-10

Figure 3 Charge build-up in nanocomposite PE at $2 \mathrm{kV}$

Figure 3(b) shows the charge formation in sample containing $10 \%$ of alumina. Charge distribution is very different from the samples containing low concentration of alumina. Positive charges are observed in the bulk of the sample with a peak close to the anode. The positive charges may be related to charge injection. This means that high concentration of alumina enhances positive charge injection significantly. From Figure 3 (a) we believe that ionisation process still occurs in the present case, however, the presence of heterocharge may be covered by dominant injected charges. Charge decay in the sample is much fast comparing with the sample containing $5 \%$ of alumina.

When the applied voltage was increased to $5 \mathrm{kV}$, charge dynamics become more complicated and the amount of charge formed in the samples becomes significant. Figure 4 shows space charge formation during the voltage application period and space charge from 'volts-off' measurements is shown here. It can be seen clearly from Figure 4 (a) that homocharge is formed close to the electrodes in sample without any alumina. This is consistent with our previous observation [10]. The amount of charge increases with the duration of the voltage application. The homocharges are closely associated with charge injection taken place from both electrodes. The amount of charge formed is similar for positive and negative charges. Charge distribution in sample PE- 1 is similar to PE-0 but the magnitude is slightly smaller. The amount of charge shows an increase with the time. There may be two possible reasons for the smaller magnitude of space charge. The first is the suppression of injection due to presence of alumina. But the result in Fig 3(b) indicates otherwise. The second explanation is the resultant effect of charge injection and ionisation. If we assume that the injection remain similar to the PE- 0 at $5 \mathrm{kV}$, the ionisation will lead to heterocharge formation in the regions adjacent to electrodes. The magnitude of hetrocharge should be increase with the amount of impurity which is associated with the level of alumina in the sample. Therefore, the presence of small amount of heterocharge will cancel out some of injected charges. The net charge present close to the electrodes will be less. PEA only shows the net charge, therefore, Figure 4 (b). When the amount of alumina is increased to $5 \%$, space charge distribution is very different as shown in Figure 4 (c). A positive peak is developed adjacent to the cathode and no significant charge buildup close to the anode. Charge distribution depends on ionisation and injection. On one hand more alumina in the sample should lead to more heterocharge formation. On the other more alumina seems to enhance charge injection, therefore, the final charge distribution is determined by the resultant effect of the two processes. In PE-5 at $5 \mathrm{kV}$, it seems that heterocharge close to the anode is balanced by injected positive charge. When more alumina is added into the LLDPE charge injection seems to dominate the charge formation as shown in Figure 4(d).

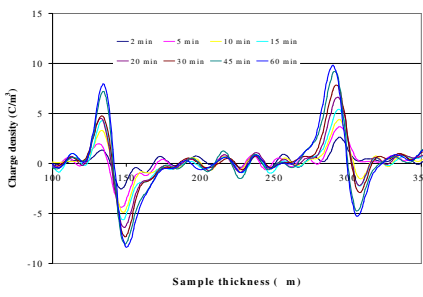

(a) PE-0

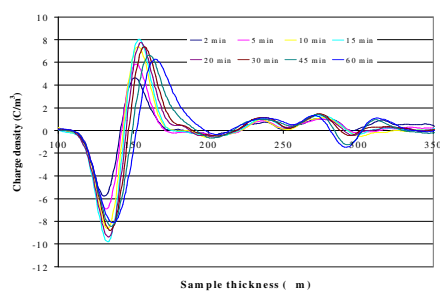

(c) PE-5

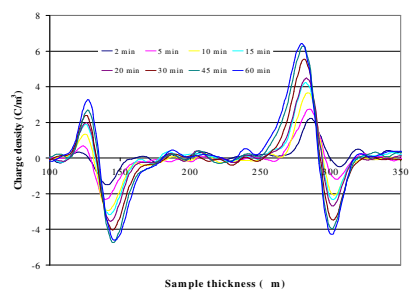

(b) PE-1

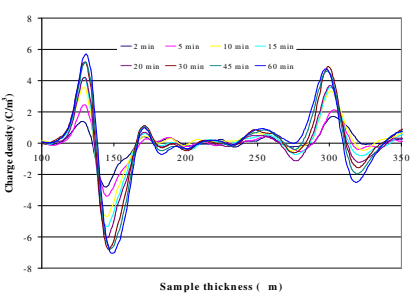

(d) PE-10
Figure 4 Charge build-up in nanocomposite PE at 5kV

When the applied voltage is increased to $10 \mathrm{kV}$ both injection and ionisation will be enhanced but probably by different extents. Figure 5 illustrates charge formation in samples containing different amount of alumina. In PE-0 charge is dominated by charge injection, i.e homocharge as 
shown in Figure 5 (a). Comparing with the PE-0 at $5 \mathrm{kV}$ the charge distribution extends towards the centre of the sample at $10 \mathrm{kV}$ but the magnitude is similar. Under the influence the applied field the injected charge tends to move towards the opposite electrode. The higher the field the more depth can charge move. The presence of the injected charge affects further injection by reducing the local electric field. At the end, a balance is reached. In PE-1 the initial charge buildup rate is slower compared with PE-0 as can be seen in Figure 5 (b). The amount of charge at the end of 60 minutes is lower. This is consistent with the results shown in Figure 4, indicating similar charge processes are in operation. When alumina is increased to $5 \%$ charge distribution becomes more complicated adjacent to the cathode. It starts with injected charge (homo) and after a few minutes, heterocharge appeared next to the homocharge and its magnitude increases with time. On the other hand, charge formation adjacent to the anode is relatively simple and dominated by injected charge. In the middle of the sample small amount of negative charge is present and this charge is believed to come from the injected charge. It seems that some of the injected negative charge is able to pass through the positive heterocharge without being recombined. In PE-10, there are more ionisable species in the sample, therefore, heterocharge appears adjacent to the two electrodes. We also know significant charge injection takes place in PE-10 especially at high fields. Consequently, two charge peaks with opposite sign in the middle of the sample are those charges injected from their respective electrodes. The amount of charge is significantly higher compared with the other samples. There are several local maximum fields across the sample. This will undoubtfully result in material deterioration and affect the electrical performance of the material. In fact, we experienced several electrical breakdowns of testing samples during 60 minutes stressing period.
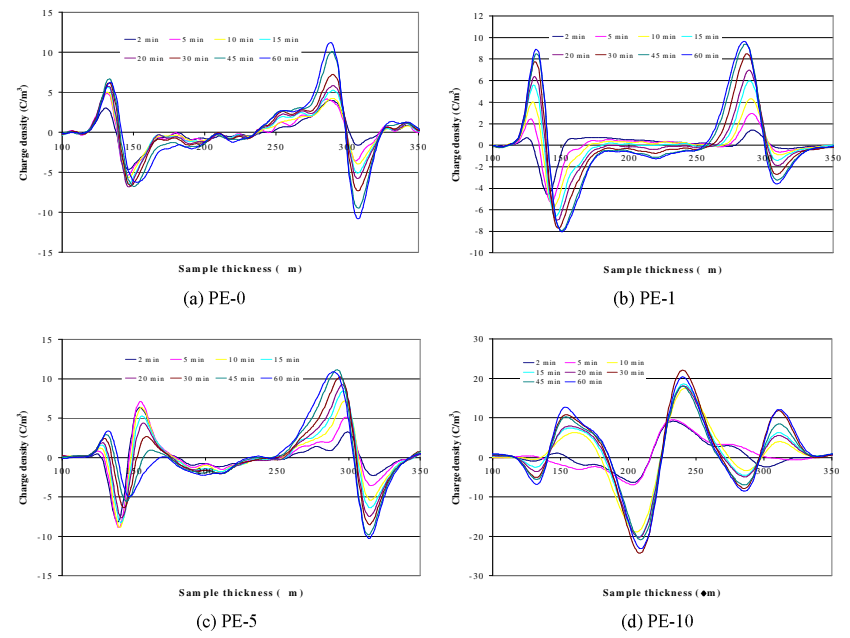

Figure 5 Charge build-up in nanocomposite PE at 10kV

\section{CONCLUSION}

The space charge formation in LLDPE loaded with different levels of nanometric sized alumina has been investigated using the PEA technique over a range of voltages. Following conclusion may be drawn from the study.

The amount of charge observed in the samples increases with the applied voltage and its duration initially but reaches to a quasi-steady state at the end of 60 minutes.

The charge formed in the sample originates from two possible sources (i) impurity ionisation from nanoparticles added and (ii) injection from two electrodes. They show slightly different relationship with the applied field, leading to a complicated charge distribution pattern with both the applied voltage and different levels of nanoparticles.

The addition of nanoparticles seems to enhance charge injection. The exact mechanisms are unknown at this stage. From space charge point of view, it can be said that more than $5 \%$ of alumina in the sample will lead to more complicated charge distribution.

\section{REFERENCES}

[1] T. J. Lewis, "Interfaces: nanometric dielectrics", Journal of Physics DApplied Physics, Vol.38, 202-212, 2005.

[2] J. K Nelson, Y. Hu, "Nanocomposite dielectrics - properties and implications", Journal of Physics D-Applied Physics, Vol.38, 213-222, 2005.

[3] M. Bohning, H. Goering, A. Fritz, K. W. Brzezinka, G. Turky, A Schonhals, B. Schartel, "Dielectric study of molecular mobility in poly(propylene-graft-maleic anhydride)/clay nanocomposites", Macromolecules, Vol. 38, 2764-2774, 2005.

[4] T. Tanaka, M. Kozako, N. Fuse, Y. Ohki, "Proposal of a multi-core model for polymer nanocomposite dielectrics", IEEE Transactions on Dielectrics and Electrical Insulation, Vol.12, 669-681. 2005

[5] Yi Yin', Jiong Chen, Jingning Yang', Dengming Xiao', Demin Tu, Rui Yin', Hongjin Qian, "Effect of Space Charge in Nanocomposite of $\mathrm{LDPE} / \mathrm{TiO}_{2}$ ", Proceedings of the $7^{\text {th }}$ Intemational Conference on Properties and Applications of Dielectric Materials, 2003 Nagoya Japan, 913-916.

[6] R. J. Fleming, T. Pawlowski, A. Ammala, P. S. Casey and K. A. Lawrence, "Electrical Conductivity and Space Charge in LDPE containing TiO2 Nanoparticles", IEEE Transactions on Dielectrics and Electrical Insulation Vol. 12, 2005, 745-753.

[7] J. K. Nelson, J. C. Fothergill, L. A. Dissado and W. Peasgood, "Towards an understanding of nanometric dielectrics" CEIDP 2002, 295-298.

[8] T. Maeno, T. Futami, H. Kushibe, T. Takada and C. M. Cooke, "Measurement of spatial charge distribution in thick dielectrics using the pulsed electroacoustic method", IEEE Trans. on EI, Vol. 23, pp. 433 - 439, 1988.

[9] G. Chen, Y. L. Chong and M. Fu, Calibration of the Pulsed Electroacoustic Technique in the Presence of Trapped Charge, Measurement Science and Technology Vol. 17, p. 974-1980, 2006.

[10] G. Chen and Z. Xu, "Space charge dynamics in low density polyethylene under dc electric fields", $12^{\text {th }}$ Intel Conf. on Electrostatics, Oxford, UK, 2007. 